Physics/Header

\title{
Exact solution of the three-boson problem at vanishing energy
}

\author{
Christophe Mora ${ }^{\mathrm{a}}$, Alexander O. Gogolin ${ }^{\mathrm{b}}$, Reinhold Egger ${ }^{\mathrm{c}}$ \\ ${ }^{a}$ Laboratoire Pierre Aigrain, ENS, Université Denis Diderot 7, CNRS; 24 rue Lhomond, 75005 Paris, France \\ ${ }^{\mathrm{b}}$ Department of Mathematics, Imperial College London, 180 Queen's Gate, London SW7 2AZ, UK \\ ${ }^{\mathrm{c}}$ Institut für Theoretische Physik, Heinrich-Heine-Universität, D-40225 Düsseldorf, Germany
}

\begin{abstract}
A zero range approach is used to model resonant two-body interactions between three identical bosons. A dimensionless phase parametrizes the three-body boundary condition while the scattering length enters the Bethe-Peierls boundary condition. The model is solved exactly at zero energy for any value of the scattering length, positive or negative. From this solution, an analytical expression for the rate of three-body recombination to the universal shallow dimer is extracted.
\end{abstract}

Key words: Few-body problem; Three-body recombination; Efimov effect

\section{Introduction}

The few-body problem is a fundamental tool in the study of dilute degenerate gases such as ultracold atomic systems [1]. It provides a basis to construct effective low energy many-body theories $[2,3,4,5,6,7]$. In addition, twoand three-body scattering properties determine the recombination and loss rates $[8,9,10,11]$ in atomic vapors and are thus of practical importance. The last decade has witnessed the rapid progress in the Feshbach resonance technique [12] which allows to tune experimentally the two-body scattering length $a$. An intense experimental and theoretical activity has then developed to investigate ultracold atoms with resonant short-ranged interactions characterized by large scattering lengths [5]. Universality [1,13] emerges in this regime, in particular at the level of few-body physics [14], for which details of the potential at short distances become unessential. In this context, universal predictions can be captured by zero range potentials where atomic interaction enters only via boundary conditions imposed on the wavefunctions.

For identical bosons, Efimov [15] has predicted a universal hierarchy of shallow bound trimer states. The corresponding energies form a geometric spectrum, which signals a discrete scaling symmetry. At unitarity, there is an infinite number of such trimers with an accumulation point at the continuum threshold. The first evidence of Efimov physics by a resonant enhancement of three-body recombination was reported in Ref. [16] with ${ }^{133}$ Cs atoms. This observation has been followed by many experiments on Efimov physics involving various atoms,

Email addresses: mora@lpa.ens.fr (Christophe Mora), a.gogolin@ic.ac.uk (Alexander O. Gogolin), egger@thphy.uni-duesseldorf.de (Reinhold Egger). 
fermionic or bosonic $[17,18,19,20,21,22,23]$. Note that the Efimov effect does not require identical bosons. For three atoms with identical mass, operators exchanging atoms commute with the Hamiltonian. The presence of a fully symmetric sector is then sufficient for the Efimov effect to develop. This rules out for instance identical fermions but it is compatible with fermions in three different spin states.

A vast literature is already devoted to the study of the three-body problem. There exist recent and comprehensive reviews $[1,13,24,25]$ on the subject covering a variety of approximate and exact techniques developed for this problem. In a remarkable series of papers, Gasaneo, Macek and Ovchinnikov have introduced the so-called Sturmian method which allows to derive some exact results in the universal, or zero range, case. They first solved a simple model [26] where only two atoms interact, and then obtained an exact solution [27] for the three-body problem at zero negative energy and for the atom-dimer scattering length. Finally, they were able to extend [28] the exact solution to an arbitrary positive energy. The zero-energy limit is feasible but remains quite involved.

For negative energies $E<0$ and a positive scattering length, the system of three bosons only exists as a single atom on one side, and a dimer on the other side binding the two remaining atoms. This atom-dimer compound is entangled at short distance and decouples at large distance. The situation changes dramatically when the zeroenergy $E=0$ threshold is crossed and a new channel opens: the three atoms can also separate freely at large distance. Therefore the zero-energy wavefunction is singular: it depends on whether the zero-energy limit is taken from positive or negative values.

In this paper we investigate the case of three identical bosons interacting via zero range potential with an arbitrary scattering length $a$. In order to proceed with a well-defined model, the Bethe-Peierls two-body boundary condition is supplemented by a three-body boundary condition. The resulting model is solved exactly at zero positive energy. As an application, an exact formula is derived for the three-body recombination rate in agreement with previous works. The approach developed in this paper has similarities with the Sturmian method of Refs. $[26,27,28]$. It is nonetheless based on an integral equation that derives from the Schrödinger equation, a method pioneered in Ref. [29]. Part of the results presented in this paper have already been shown in a preceding letter [30]. The zero-range model is introduced and reviewed in Sec. 2. The solution of the three-boson problem at zero energy is explicited in Sec. 3 both for positive and negative scattering lengths. It is used in Sec. 4 to derive the rate for three-body recombination. Sec. 5 summarizes the results obtained in this paper.

\section{Zero-range model}

\subsection{Integral equation}

We introduce a zero-range model for three identical bosons of mass $m$. The three-boson wavefunction is simply the solution of the free Schrödinger equation with, in addition, the Bethe-Peierls boundary condition

$$
\left.\psi(\mathbf{x}, \mathbf{y})\right|_{y \rightarrow 0}=\frac{f(\mathbf{x})}{4 \pi}\left(\frac{1}{y}-\frac{1}{a}\right),
$$

which recovers the two-body physics. $\mathbf{y}$ is the distance between two bosons (denoted 1 and 2 ) and $\sqrt{3} \mathbf{x} / 2$ the distance between their centroid and the third boson (denoted 3), the center of mass being decoupled. The two other two-body boundary conditions follow from symmetrization of the wavefunction. The Schrödinger equation on $\psi$ can be transformed into an integral equation for the reduced atom-dimer function $f(\mathbf{x})$. The corresponding procedure is now standard $[6,7,11,29,31,32,33]$ and we will only briefly review it here. Applying the Laplacian on the three boundary conditions implied from Eq. (1), the Schrödinger equation acquires a source term

$$
-\left(\nabla_{\mathbf{x}}^{2}+\nabla_{\mathbf{y}}^{2}+\frac{m E}{\hbar^{2}}\right) \psi(\mathbf{x}, \mathbf{y})=\mathcal{S}(\mathbf{x}, \mathbf{y})
$$

with $\mathcal{S}(\mathbf{x}, \mathbf{y})=(1+\hat{Q}) f(\mathbf{x}) \delta(\mathbf{y})$. The operator $\hat{Q}=\hat{P}_{23}+\hat{P}_{13}$, where $\hat{P}_{i j}$ exchanges atoms $i$ and $j$, ensures the proper bosonic symmetry.

Using the Green's function

the solution of Eq. (2) takes the form

$$
G_{\varepsilon}\left(\mathbf{p}_{1}, \mathbf{p}_{2}\right)=\frac{1}{p_{1}^{2}+p_{2}^{2}-\varepsilon}
$$

$$
\psi(\mathbf{x}, \mathbf{y})=\psi_{0}(\mathbf{x}, \mathbf{y}) \theta(E)+\int d^{3} x^{\prime} d^{3} y^{\prime} G_{m E / \hbar^{2}}\left(\mathbf{x}-\mathbf{x}^{\prime}, \mathbf{y}-\mathbf{y}^{\prime}\right) \mathcal{S}\left(\mathbf{x}^{\prime}, \mathbf{y}^{\prime}\right)
$$


where $\psi_{0}$, a symmetrized combination of plane waves, describes the incoming wave. $\psi_{0}$ is solution of the homogeneous part of Eq. (2). $\theta(E)$ is the Heavyside function. The integral equation is closed on the function $f$ by taking the $y \rightarrow 0$ limit in Eq. (4) with the Bethe-Peierls boundary condition (1). This results in

$$
\left(\hat{L}_{m E / \hbar^{2}}-\frac{1}{a}\right) f(\mathbf{x})=4 \pi \psi_{0}(\mathbf{x}, 0) \theta(E)
$$

where the expression of the operator $\hat{L}_{\varepsilon}$ is given in momentum space by

$$
\hat{L}_{\varepsilon} f(\mathbf{k})=\sqrt{-\varepsilon+k^{2}} f(\mathbf{k})-\frac{2}{\sqrt{3} \pi^{2}} \int d^{3} k^{\prime} \frac{f\left(\mathbf{k}^{\prime}\right)}{k^{2}+k^{\prime 2}+\mathbf{k} \cdot \mathbf{k}^{\prime}-3 \varepsilon / 4} .
$$

\subsection{Three-body boundary condition}

The integral equation (5) was first derived by Skorniakov and Ter-Martirosian [29], and shown later by Danilov [34] to be ill-defined with a dense and unbounded spectrum at negative energy. This unphysical prediction is related to the Thomas collapse[35], or fall to the center effect, at vanishing distances between the three bosons. It suggests that an additional boundary condition [36,37] is necessary if we wish to define a proper zero-range model for this problem. The operator $\hat{L}_{\varepsilon}$ does not break the rotational symmetry and thus decouples the different angular momenta. We shall concentrate on the rotationally invariant s-wave channel, $f(\mathbf{x})=f(x)$, where the fall to the center pathology does occur. At small distances $x \rightarrow 0$, the inverse scattering length $1 / a$, the energy $\varepsilon$ and the incoming wavefunction $\psi_{0}$ can be neglected in Eq.(5). Therefore all wavefunctions become solutions of the universal homogeneous equation

$$
\hat{L}_{0} f(x)=0 .
$$

The operator $\hat{L}_{0}$ has a simple action on power functions (see appendix A),

$$
\hat{L}_{0} x^{-(1+\nu)}=\phi(\nu) x^{-(2+\nu)} \quad \phi(\nu)=\frac{\nu}{\tan (\pi \nu / 2)} G(\nu)
$$

where the function

$$
G(\nu)=1-\frac{8}{\sqrt{3}} \frac{\sin (\nu \pi / 6)}{\nu \cos (\nu \pi / 2)}
$$

has been defined. $G(\nu)$ has an infinite number of roots on the real axis and two complex conjugate imaginary roots at $\nu= \pm i s_{0}$ with $s_{0} \simeq 1.00624$. They correspond to the incoming and outgoing solutions to Eq. (7), $\frac{e^{ \pm i s_{0}} \ln x}{x}$, that dominate the $x \rightarrow 0$ asymptotic behavior of $f$. All other solutions to Eq. (7) attenuate more rapidly as $x$ tends to zero. The limiting form of $f(x)$ is thus not fully determined since any combination of these two complex conjugate solutions is admissible. This has been shown [34] to lead to an unphysical dense and unbounded spectrum: there exists an eigenstate for any (negative) value of the energy $E$.

The three-body problem becomes well-defined if one imposes the additional boundary condition $[15,34,36]$

$$
\left.f(x)\right|_{x \rightarrow 0} \propto \frac{\sin \left[s_{0} \ln (x / \ell)+\varphi\right]}{x},
$$

for the asymptotic behaviour at vanishing $x$, i.e. when the three bosons all coincide. The length $\ell$ does not bear any physical meaning, its only purpose is to set up a reference length in the logarithm. It is defined up to a multiplicative factor $e^{\pi / s_{0}}$ and can therefore take arbitrarily small or large values. All possible boundary conditions are exhausted when $\varphi$ is taken in the interval $[0, \pi]$, and changing the value of $\ell$ only amounts to shift the origin of $\varphi$. Note that $\varphi$ has a clear physical meaning: it can be thought of as the phase shift between the incoming and outgoing waves with respect to the origin $x=0$. The amplitudes of these two waves are equal as a result of flux conservation. This property is however violated once the recombination to deep dimer states is included in the formalism $[38,39]$.

Eq. (10) can be given a transparent physical meaning. The phase shift $\varphi$ is determined by short-range three-body physics occurring at inter-particles distances for which the zero-range approach is not valid. This short-distance physics is not sensitive to the larger length scales of the problem - such as the scattering length $a-$ and $\varphi$ appears as a universal phase that applies to all eigenstates of the three-body problem. $\varphi$ thus plays the role of a threebody parameter. It is the analogue of the scattering length $a$ in the two-body problem. Finally, Eq. (10) exhibits a discrete scaling symmetry $x \rightarrow x e^{\pi / s_{0}}$ from which the geometrical pattern of Efimov states originates. 


\subsection{Two-channel model}

For a model with a finite range potential of size $\ell_{0}$, the scaling limit is such that $\ell_{0}$ is sent to zero while the scattering length $a$ is kept finite [1]. A zero range model is expected to describe directly the scaling limit of a more realistic finite range potential. Enforcing a particular set of boundary conditions to the solutions of the free Schrödinger equation does not automatically constitute a well-defined zero range model. It is indeed notoriously difficult to prove rigorously that the corresponding model is self-adjoint. We have circumvented these difficulties in Ref. [30] by introducing a two-channel model: interactions between atoms in an open channel are mediated by a molecular state in a closed channel. In addition to the scattering length $a$, this model introduces an additional length $R^{*}$ that corresponds to the size of the molecular state coupled to the open channel continuum. Similarly to Refs. [11,40], it amounts to give some energy dependence to the scattering length $a$, and the length $R^{*}$ is proportional to the s-wave effective range [1]. In addition to providing a quantitative description of a narrow Feshbach resonance, the two-channel model can also be seen as a toy model describing a generic two-body interaction in the scaling limit. It is moreover self-adjoint by construction and thus regularizes the pathologies associated with the three-body problem.

The scaling limit $R^{*} \rightarrow 0$ of the two-channel model introduced in Ref. [30] can be checked to reproduce the boundary conditions Eqs. (1) and (10). Within the two-channel model with $R^{*} \rightarrow 0$, Eq. (5) is replaced by

$$
\left(-R^{*} \nabla_{\mathbf{x}}^{2}+\hat{L}_{\varepsilon}-\frac{1}{a}\right) f(\mathbf{x})=4 \pi \psi_{0}(\mathbf{x}, 0) \theta(E)
$$

where $\varepsilon=m E / \hbar^{2}$. Since $R^{*} \ll a$, this equation can be split into two integral equations. For $x \gg R^{*}$, the term $\propto R^{*}$ inside the parenthesis can be neglected and $f(\mathbf{x})$ is solution of Eq. (5). On the other hand, for $x \ll a, 1 / \sqrt{|\varepsilon|}$, all $a$ and $\varepsilon$ dependences disappear and Eq. (11) simplifies to

$$
\left(-R^{*} \nabla_{\mathbf{x}}^{2}+\hat{L}_{0}\right) f(\mathbf{x})=0 .
$$

The complete solutions to Eq. (11) are obtained by matching the asymptotes of the different solutions in the region $R^{*} \ll x \ll a$, where the validity domains of Eqs. (5) and (12) overlap and Eq. (7) holds. In other words, Eq. (12) serves as a boundary condition for the solutions of Eq. (5). As detailed in appendix A, the solution to Eq. (12) is obtained following Ref. [30]. For $R^{*} \ll x \ll a$, one recovers the asymptotic form (10) with $R_{0} \equiv$ $\ell e^{-\varphi / s_{0}} \simeq 0.577 R^{*}$.

\section{Zero energy solution}

Having established a well-defined zero-range approach for three bosons, we proceed with the construction of the solution at zero (positive) energy. We shall solve Eq. (5) at $E=0^{+}$with the boundary condition (10).

The incoming wave is generally a symmetrized combination of plane waves,

$$
\psi_{0}\left(\mathbf{r}_{1}, \mathbf{r}_{2}, \mathbf{r}_{3}\right)=\frac{1}{\sqrt{6} V^{3 / 2}} \sum_{\sigma} P_{\sigma} e^{i \sum_{i=1}^{3} \mathbf{k}_{i} \cdot \mathbf{r}_{i}}
$$

where the summation includes all permutations $P_{\sigma}$ of $\{1,2,3\} . V$ is the system volume and the wavefunction has been normalized to one. All wavevectors $\mathbf{k}_{i}$ vanish at zero energy, hence $\psi_{0}(\mathbf{x}, 0)=\sqrt{6 / V^{3}}$. Let us define the auxiliary function $\tilde{f}(x)$ such that

$$
f(x)=-4 \pi a \sqrt{\frac{6}{V^{3}}}\left(1-\frac{4}{\sqrt{3}} \frac{a}{x}+\frac{4}{\sqrt{3}} \phi(0) \tilde{f}(x)\right),
$$

where $\phi(\nu)$ is defined Eq. (8). In particular, one has $\phi(-1)=-4 / \sqrt{3}$ and $\phi(0)=\frac{2}{\pi} G(0)=\frac{2}{\pi}[1-4 \pi /(3 \sqrt{3})]$. The property (8) of the operator $\hat{L}_{0}$ on power functions yields the following integral equation

$$
\left(\hat{L}_{0}-\frac{1}{a}\right) \tilde{f}(\mathbf{x})=\frac{a}{x^{2}}
$$

An exact solution to this equation is given in the next four subsections. Subsections 3.1, 3.2 and 3.3 are devoted to the case of a positive scattering length $a>0$, while the case $a<0$ is also exactly solved in Subsection 3.4. An alternative way of expressing the solutions to Eq. (14) has been proposed in Ref. [41]. 


\subsection{Solutions of the homogeneous equation}

We consider the case $a>0$. The homogeneous equation associated to Eq. (14) corresponds to replace the right-hand-side of Eq. (14) by zero. The solution to this integral equation can be written as a Barnes-type contour integral

$$
\beta_{1}^{ \pm}(x)=\frac{a}{x} \int_{-i \infty}^{+i \infty} \frac{d \nu}{2 i \pi} C_{ \pm}(\nu) \Gamma(\nu) \sin (\pi \nu / 2)\left(\frac{x}{a}\right)^{-\nu}
$$

with the Gamma function $\Gamma(z)$. The integration contour runs on the right of the imaginary axis with a small and positive real part. $C_{ \pm}$is a set of two functions to be determined. We apply $\hat{L}_{0}$ to Eq. (15), and then use the identities $\nu \Gamma(\nu)=\Gamma(\nu+1), \cos (\pi \nu / 2)=\sin [\pi(\nu+1) / 2]$ and Eq. (8) to obtain

$$
\hat{L}_{0} \beta_{1}^{ \pm}(x)=\frac{1}{x} \int_{-i \infty}^{+i \infty} \frac{d \nu}{2 i \pi} G(\nu) C_{ \pm}(\nu) \Gamma(\nu+1) \sin [\pi(\nu+1) / 2]\left(\frac{x}{a}\right)^{-(\nu+1)} .
$$

Assuming the identity

$$
C_{ \pm}(\nu+1)=G(\nu) C_{ \pm}(\nu)
$$

and the property that $C_{ \pm}(\nu)$ has no singularities (poles) within the stripe $0<\operatorname{Re}(\nu)<2$ of the complex plane, the integral contour can be shifted back, $\nu \rightarrow \nu-1$, with the required result $\hat{L}_{0} \beta_{1}^{ \pm}(x)=\beta_{1}^{ \pm}(x) / a$. Using the Weierstrass theorem, $G(\nu)$ can be expressed in terms of its poles $\pm b_{p}$, where $b_{p}=2 p+1(p \in \mathrm{N})$ and its zeros $\pm u_{p}$ in the complex plane, namely

$$
G(\nu)=\prod_{n=0}^{+\infty} \frac{\nu^{2}-u_{p}^{2}}{\nu^{2}-b_{p}^{2}}
$$

There is an infinite number of zeros on the real axis, with $u_{1}=4, u_{2} \simeq 4.6 \ldots$, and exactly two on the imaginary axis, $u_{0}= \pm i s_{0}$. For $p \gg 1$, we find the analytical estimate

$$
u_{p}=b_{p}+\frac{8}{\pi \sqrt{3}(2 p+1)} \times\left\{\begin{array}{c}
2, \quad \bmod (2 p+1,6)=3, \\
-1, \bmod (2 p+1,6)=1,5 .
\end{array}\right.
$$

Using the representation (18) and the aforementioned identity $\nu \Gamma(\nu)=\Gamma(\nu+1)$, it can be verified that the two functions

$$
C_{ \pm}(\nu)=\prod_{p=0}^{\infty} \frac{\Gamma\left(\nu+u_{p}\right) \Gamma\left(1-\nu+b_{p}\right)}{\Gamma\left(\nu+b_{p}\right) \Gamma\left(1-\nu+u_{p}\right)}
$$

with $u_{0}= \pm i s_{0}$ respectively, are solutions of the identity (17) with no singularity in the stripe $0<\operatorname{Re}(\nu)<2$. To be more specific, the closest singularities from the forbidden stripe are for $\nu=2$ and $\nu=\mp i s_{0}$ for the functions $C_{ \pm}(\nu)$ respectively. One also verifies that the integral in Eq. (15) is well-defined with Eq. (20), i.e. the integrand is integrable. In fact, it is physically expected that the two functions (20) exhaust the set of functions that verify the requirements: (i) integrability, (ii) absence of poles in the forbidden stripe and, (iii) solution of the identity (17). Remarkably, Ref. [27] has introduced an alternative expression for the function $C_{ \pm}(\nu)$. The equivalence with the definition used here is not straightforward to show.

To summarize our findings, we have exhibited two independent solutions of the homogeneous equation corresponding to Eq. (14) for $a>0$. They are given by Eq. (15) with Eq. (20).

\subsection{Asymptotic behaviour at small $x$ for $\beta_{1}^{ \pm}(x)$}

As we shall see below, the solutions $\beta_{1}^{ \pm}(x)$ give rise to the incoming and outgoing asymptotes $e^{ \pm i s_{0} \ln x} / x$ as $x \rightarrow 0$. The proper combination of $\beta_{1}^{+}$and $\beta_{1}^{-}$is therefore necessary to match the boundary condition (10).

The asymptotic behaviour for $x \ll a$ is obtained by closing the integration contour on the left with a semi-circle at infinity in the left half plane. The pole at $\nu=\mp i s_{0}$ gives the dominant contribution with the result

$$
\beta_{1}^{+}(x) \simeq \frac{a}{i x} \sinh \left(\frac{\pi s_{0}}{2}\right)\left|\operatorname{Res}\left(C_{+},-i s_{0}\right) \Gamma\left(-i s_{0}\right)\right| e^{i\left[\delta_{0}+s_{0} \ln (x / a)\right]} \propto \frac{e^{i s_{0} \ln x}}{x}, \quad \beta_{1}^{-}(x)=\left[\beta_{1}^{+}(x)\right]^{*}
$$

where $\delta_{0}$ is the phase of $\operatorname{Res}\left(C_{+},-i s_{0}\right) \Gamma\left(-i s_{0}\right)$ and $\operatorname{Res}\left(C_{+},-i s_{0}\right)$ denotes the residue of $C_{+}(\nu)$ at $\nu=-i s_{0}$. Using the result (B.2) of appendix B, the phase $\delta_{0}$ can be written $\delta_{0}=\pi \gamma+\operatorname{Arg} \Gamma\left(-i s_{0}\right) \simeq 1.5875[27]$. Here $\gamma$ is

$$
\gamma=-\frac{1}{2}-\frac{1}{\pi} \operatorname{Arg} C_{+}\left(i s_{0}\right) \simeq-0.090518155
$$


correcting a typo in Eq. (17) of Ref. [30]. The linear combination $\beta_{1}=e^{-i \delta_{1}} \beta_{1}^{+}+e^{i \delta_{1}} \beta_{1}^{-}$, with

$$
\delta_{1}=\delta_{0}+s_{0} \ln \left(R_{0} / a\right)=\pi \gamma+\operatorname{Arg} \Gamma\left(-i s_{0}\right)+s_{0} \ln \left(R_{0} / a\right),
$$

and $R_{0} \equiv \ell e^{-\varphi / s_{0}}$, recovers the three-body boundary condition (10).

\subsection{Complete solution for $a>0$}

A solution to Eq. (14) is derived using a Barnes-type integral similar to the homogeneous case. It reads

$$
\beta_{2}(x)=\mathcal{B}\left(e^{-i \delta_{1}} \beta_{2}^{+}(x)-e^{i \delta_{1}} \beta_{2}^{-}(x)\right),
$$

with

$$
\mathcal{B}=\frac{\pi}{2 i \cos \delta_{1} \sqrt{-G(0)}}
$$

The phase $\delta_{1}$ is defined Eq. (23), and

$$
\beta_{2}^{ \pm}(x)=\frac{a}{x} \int_{-i \infty}^{+i \infty} \frac{d \nu}{2 i \pi} C_{ \pm}(\nu) \Gamma(\nu) \frac{\sin (\pi \nu / 2)}{\tan (\pi \nu)}\left(\frac{x}{a}\right)^{-\nu}
$$

with the functions $C_{ \pm}(\nu)$ given Eq. (20). Before expliciting this solution, it can be checked that the boundary condition (10) is reproduced in the limit $x \ll a$. Similarly to Sec. 3.2, the integral contour in Eq. (26) can be closed around the left half plane. The asymptotic form is dominated by the closest poles located at $\nu= \pm i s_{0}$ and $\nu=0$. The contribution from the $\nu=0$ pole is easily computed. Using the property $C_{ \pm}(\nu) C_{ \pm}(1-\nu)=1$ and Eq. (B.5) derived in appendix B, it is shown to cancel exactly the term $\propto 1 / x$ in Eq. (13) when $f(x)$ is expressed in terms of $\beta_{2}(x)$. As a result, the asymptotic form for $f(x)$ is solely determined by the poles at $\nu= \pm i s_{0}$. Following Sec. 3.2, the contributions of these two poles recover the behaviour (10).

In order to verify Eq. (24), we apply $\hat{L}_{0}$ to Eq. (26). The result is

$$
\hat{L}_{0} \beta_{2}^{ \pm}(x)=\frac{1}{x} \int_{-i \infty+1}^{+i \infty+1} \frac{d \nu}{2 i \pi} C_{ \pm}(\nu) \Gamma(\nu) \frac{\sin (\pi \nu / 2)}{\tan (\pi \nu)}\left(\frac{x}{a}\right)^{-\nu}
$$

where the contour lies slightly on the right of the line $\operatorname{Re}(\nu)=1$. The main difference with the homogeneous case of Sec. 3.1 is that the integrand now has a pole in the stripe $0<\operatorname{Re}(\nu)<2$. This pole is located at $\nu=1$ due to the $\tan (\pi \nu)$ in the denominator. The integral contour can nevertheless be shifted back, $\nu \rightarrow \nu-1$, with the result $\beta_{2}^{ \pm}(x) / a$ and an additional contribution that can be evaluated from the pole's residue at $\nu=1$, namely

$$
\hat{L}_{0} \beta_{2}^{ \pm}(x)=\frac{\beta_{2}^{ \pm}}{a}+\frac{C_{ \pm}(1)}{\pi} \frac{a}{x^{2}},
$$

where $C_{ \pm}(1)= \pm i \sqrt{-G(0)}$ from Eq. (B.5) in appendix B. Using Eq. (28), the function $\beta_{2}(x)$ from Eq. (24) is found to be solution of the integral Eq. (14).

To summarize, the general solution to the integral equation $(14)$ is of the form $\tilde{f}(x)=\beta_{2}(x)+\mathcal{C} \beta_{1}(x)$, where $\mathcal{C}$ is an arbitrary complex coefficient.

\subsection{Exact solution for $a<0$}

We finally discuss the case of negative scattering length $a<0$. In that case, the operator $\hat{L}_{0}+1 /|a|$ does not have a homogeneous solution and can be inverted. The physical meaning of this property is that (universal) dimers do not form for $a<0$. The atom-dimer sector is therefore absent at large distances between the atoms. Eq. (14), with the boundary condition (10), possesses a unique solution, namely

$$
\beta_{3}(x)=\mathcal{B}\left(e^{-i \delta_{1}} \beta_{3}^{+}(x)-e^{i \delta_{1}} \beta_{3}^{-}(x)\right),
$$

with $\mathcal{B}$ given Eq. (25) and

$$
\beta_{3}^{ \pm}(x)=-\frac{|a|}{x} \int_{-i \infty}^{+i \infty} \frac{d \nu}{2 i \pi} C_{ \pm}(\nu) \Gamma(\nu) \frac{\sin (\pi \nu / 2)}{\sin (\pi \nu)}\left(\frac{x}{|a|}\right)^{-\nu}
$$


Similarly to Sec. (3.3), the boundary condition (10) is verified by closing the contour around the left hand plane and evaluating the residues at $\nu=0$ and $\nu= \pm i s_{0}$. Applying $\hat{L}_{0}$ to $\beta_{3}$ amounts to shift the integral contour $\nu \rightarrow \nu+1$ with a sign change. A pole at $\nu=1$ is encountered when the contour is shifted back, leading to

$$
\hat{L}_{0} \beta_{3}^{ \pm}(x)=-\frac{\beta_{3}^{ \pm}}{|a|}-\frac{C_{ \pm}(1)}{\pi} \frac{|a|}{x^{2}} .
$$

This result, inserted into Eq. (29), shows that $\beta_{3}(x)$ is solution of Eq. (14) for $a<0$.

\section{Rate of three-body recombination}

Three-body recombination is a collision process in which three incoming atoms form a (dimer) molecule and an atom. The binding energy of the dimer is converted into kinetic energy, and recombination thus produces losses of atoms from the trap in ultracold atomic vapors. This effect is often important as it can limit experimentally the lifetime of quantum gases. It also offers a convenient experimental tool to probe resonances in the (few)threebody problem that are usually accompanied by increasing recombination [42] and therefore loss rate. In the case of resonant interactions (large $a>0$ ), the shallow dimer has universal features and recombination can be quantitatively captured by a zero-range model such as the one discussed in this paper.

\subsection{Formalism and result for the recombination rate}

The general solution $\tilde{f}(x)=\beta_{2}(x)+\mathcal{C} \beta_{1}(x)$, that was derived in Sec. 3.3, can be inserted in Eq. (13) to find $f(x)$, which gives access to the complete three-boson wavefunction $\psi(\mathbf{x}, \mathbf{y})$ via Eq. (4). The resulting expression is cumbersome and we shall not write it here. Instead, the asymptotic properties of $\psi$ can be discussed quite generally. Two relevant sectors emerge upon considering the large distance asymptotes. Sector I: for $x \rightarrow+\infty$ and $y \rightarrow+\infty$, the wavefunction corresponds to three free atoms in a symmetrized combination of plane waves. Sector II: the second sector splits itself into three equivalent domains. Taking $x \rightarrow+\infty$ with $y$ fixed describes the atom 3 and a dimer, formed by atoms 1 and 2, flying apart. The two remaining domains are obtained by exchanging the single atom 3 by 1 or 2 .

For both sectors, there are incoming and outgoing waves whose relative coefficients depend on the constant $\mathcal{C}$. In order to calculate the three-body recombination rate, the following scattering situation is considered: the incoming wave is formed solely by three free atoms and the flux probability to leak into the three domains of the atom-dimer sector is computed. The constant $\mathcal{C}$ is therefore chosen in order to cancel the atom-dimer incoming wave.

We use the notation $r=\sqrt{3} x / 2$ for the distance between the atom 3 and the 1-2 centroid. For $x \rightarrow+\infty$ with $y$ fixed, the asymptotical form $\psi(\mathbf{x}, \mathbf{y}) \simeq \phi_{0}(y) \phi_{\text {ad }}(r)$ is expected, where $\phi_{0}(y)=e^{-y / a} /(y \sqrt{2 \pi a})$ is the normalized two-body bound state (dimer) wavefunction, and

$$
\phi_{\text {ad }}(r)=\mathcal{A} \sqrt{\frac{a}{8 \pi}} \frac{\sqrt{3}}{2} \frac{e^{i \frac{2}{\sqrt{3}} r / a}}{r},
$$

where $\mathcal{A}$ is a constant that will be extracted from the exact solution. The wavefunction $\phi_{\text {ad }}(r)$ describes the atomdimer relative motion corresponding to an outgoing scattered wave by the recombination process. The $y \rightarrow 0$ limit in the asymptotical form of $\psi(\mathbf{x}, \mathbf{y})$ can be compared to the Bethe-Peierls boundary condition (1) to extract $f$ with the result

$$
f(x) \simeq \mathcal{A} \frac{e^{i x / a}}{x}
$$

in agreement with the behaviour of $f(x)$ at large $x$ that shall be derived in Sec. 4.2. Interestingly, this result shows that the large $x$ study of $f(x)$ is sufficient to determine the wavefunction in the atom-dimer sector through the knowledge of $\mathcal{A}$. A similar reasoning also indicates that a vanishing atom-dimer incoming wave is equivalent to a vanishing $e^{-i x / a} / x$ term in $f(x)$ at large $x$.

Eq. (32) can be used to compute the rate of three-body recombination. The current probability associated to $\phi_{\text {ad }}(r)$ is given by $j(r)=\frac{\hbar}{2 i(2 m / 3)}\left[\phi_{\text {ad }}^{*}(r) \partial_{r} \phi_{\text {ad }}(r)-\partial_{r} \phi_{\text {ad }}^{*}(r) \phi_{\text {ad }}(r)\right]$ where $2 m / 3$ is the atom-dimer reduced mass. The probability flux $\Phi_{i}$ to leave the scattering region in one of the atom-dimer domains ( $i$ denotes the indices of 
the single atom) can be obtained by integrating $j(r)$ over a sphere with an infinitely large radius. An additional integration over the free center of mass position multiplies by the volume $V$ and one finds

$$
\frac{\Phi}{3}=\Phi_{i}=V \lim _{r \rightarrow+\infty} 4 \pi r^{2} j(r)=\frac{3 \sqrt{3}}{8} \frac{\hbar V}{m}|\mathcal{A}|^{2},
$$

where $\Phi$ denotes the total flux to the atom-dimer sector, or sector II. It is also possible to relate this flux to the large $x$ form of the auxiliary function $\tilde{f}$ defined Eq. (13), namely

$$
\begin{aligned}
\tilde{f}(x) & =\tilde{\mathcal{A}} \frac{e^{i x / a}}{x}, \\
\Phi_{i} & =192 \sqrt{3} \pi^{2} a^{2} \phi^{2}(0) \frac{\hbar}{m} \frac{|\tilde{\mathcal{A}}|^{2}}{V^{2}},
\end{aligned}
$$

where $\phi(\nu)$ is defined Eq. (8) and $\phi(0)=\frac{2}{\pi} G(0)=\frac{2}{\pi}[1-4 \pi /(3 \sqrt{3})]$. The value of $\tilde{\mathcal{A}}$ can be extracted from the exact solution of Eq. (14) derived in Sec. 3.3. This calculation is postponed to Sec. 4.2.

For a dilute gas of $N$ bosons with density $n=N / V$, the three-body recombination rate $\alpha_{\text {rec }}$ is defined [1] such that the number of recombination events per time and per volume is $\alpha_{\text {rec }} n^{3}$. Hence,

$$
\alpha_{\text {rec }}\left(\frac{N}{V}\right)^{3}=\frac{1}{V} \frac{d N_{\text {rec }- \text { events }}}{d t}=\frac{\Phi}{V} \frac{N(N-1)(N-2)}{6} \simeq \frac{\Phi}{V} \frac{N^{3}}{6}
$$

where $\frac{N(N-1)(N-2)}{6}$ is the number of triplets among the $N$ bosons. The resulting expression for the three-body recombination rate is

$$
\alpha_{\text {rec }}=96 \sqrt{3} \pi^{2} a^{2} \phi^{2}(0) \frac{\hbar}{m}|\tilde{\mathcal{A}}|^{2}
$$

\subsection{Wavefunction at large $x$}

For $x \gg a$, the integrands in the expressions of $\beta_{1}(x)$ and $\beta_{2}(x)$, Eqs. (15) and (24), develop rapid oscillations. The asymptotical behaviour can thus be deduced from a saddle-point analysis that is detailed in appendix C. Focusing on the exact solution,

$$
\tilde{f}(x)=\beta_{2}(x)+\mathcal{C} \beta_{1}(x)=\mathcal{B}\left(e^{-i \delta_{1}} \beta_{2}^{+}(x)-e^{i \delta_{1}} \beta_{2}^{-}(x)\right)+\mathcal{C}\left(e^{-i \delta_{1}} \beta_{1}^{+}(x)+e^{i \delta_{1}} \beta_{1}^{-}(x)\right),
$$

the incoming atom-dimer wave $\left(\propto e^{-i x / a} / x\right)$ is seen to vanish with the choice

$$
\mathcal{C}=\frac{\mathcal{B}}{i} \frac{1-e^{-2 \pi s_{0}} e^{-2 i \delta_{1}}}{1+e^{-2 \pi s_{0}} e^{-2 i \delta_{1}}}
$$

Inserting this result back into the large $x \gg a$ expression of $\tilde{f}(x)$, Eq. (35a) is recovered with the coefficient

$$
\tilde{\mathcal{A}}=\mathcal{B} e^{i\left(\delta_{\infty}-\delta_{1}\right)} \frac{a e^{\pi s_{0}}}{2}\left(1-e^{-2 \pi s_{0}} e^{2 i \delta_{1}}\right)\left[1-i \frac{\mathcal{C}}{\mathcal{B}} \frac{1-e^{-2 \pi s_{0}} e^{2 i \delta_{1}}}{1+e^{-2 \pi s_{0}} e^{2 i \delta_{1}}}\right] .
$$

Some simple algebraic manipulations further lead to the expression

$$
\tilde{\mathcal{A}}=\mathcal{B} e^{i\left(\delta_{\infty}-\delta_{1}\right)} \frac{4 a}{i} \frac{\sin \delta_{1} \cos \delta_{1}}{e^{\pi s_{0}}+e^{-\pi s_{0}} e^{-2 i \delta_{1}}} .
$$

The identity

$$
\frac{1-e^{-2 i \delta_{1}}}{1+e^{-2 \pi s_{0}} e^{-2 i \delta_{1}}}=\frac{1-e^{-2 i \delta_{r}}}{1-e^{-2 \pi s_{0}}}
$$

with the phase $\delta_{r}=\delta_{1}-\operatorname{Arg}\left(1+e^{-2 \pi s_{0}} e^{2 i \delta_{1}}\right)$ is used together with the value of $\mathcal{B}$ from Eq. (25) to derive

$$
\tilde{\mathcal{A}}=-\frac{\pi a}{\sqrt{-G(0)}} e^{i\left(\delta_{\infty}-\delta_{r}\right)} \frac{\sin \delta_{r}}{\sinh \left(\pi s_{0}\right)}
$$

The rate for three-body recombination is finally given by

$$
\alpha_{\mathrm{rec}}=\frac{128 \pi^{2}(4 \pi-3 \sqrt{3})}{\sinh ^{2}\left(\pi s_{0}\right)} \frac{\hbar^{2} a^{4}}{m} \sin ^{2}\left(\delta_{r}\right) .
$$

This function shows periodic oscillations as a function of $\ln \left(R_{0} / a\right)$, with a period given by the scaling factor $e^{\pi s_{0}}$, and a dimensionless amplitude $128 \pi^{2}(4 \pi-3 \sqrt{3}) / \sinh ^{2}\left(\pi s_{0}\right) \simeq 67.1177$. In practice, $e^{-2 \pi s_{0}} \simeq 0.0012$ is a very small number such that $\delta_{1} \simeq \delta_{r}$ and the oscillations are almost sinusoidal. 
Instead of Eq. (44), an alternative formula can be obtained for the three-body recombination rate by taking directly the absolute value squared of Eq. (41) and using that $\left|e^{\pi s_{0}}+e^{-\pi s_{0}} e^{-2 i \delta_{1}}\right|^{2}=4 \sinh ^{2}\left(\pi s_{0}\right)+4 \cos ^{2}\left(\delta_{1}\right)$. The result reads

$$
\alpha_{\mathrm{rec}}=\frac{128 \pi^{2}(4 \pi-3 \sqrt{3})}{\sinh ^{2}\left(\pi s_{0}\right)} \frac{\hbar^{2} a^{4}}{m} \frac{\sin ^{2}\left(\delta_{1}\right) \sinh ^{2}\left(\pi s_{0}\right)}{\sinh ^{2}\left(\pi s_{0}\right)+\cos ^{2}\left(\delta_{1}\right)},
$$

which coincides with Eq. (44) and recovers the result of Refs. [28,30,43,41]. Ref. [13] has pointed out that the expression (44) also derives from the use of the optical theorem and the knowledge of the elastic boson-diboson scattering solution at zero (negative) energy determined in Ref. [27].

\section{Conclusion}

We have solved exactly the wavefunction at vanishing energy of three identical bosons with zero range interactions. The zero range model requires a set of two boundary conditions. The first one is the standard Bethe-Peierls condition when two atoms meet. An additional three-body boundary condition when the three atoms coalesce is then necessary. It is due to the fall to the center effect, also responsible for the emergence of the Efimov bound states. It is characterized by a dimensionless parameter $\varphi$ which describes the phase shift between the outgoing and the incoming waves toward the region where the three atoms coincide. Close to this region, the wavefunction displays oscillation as a function of $\ln (R / a)$ where $R$ denotes the hyperradius of the three bosons. These logarithmic oscillations thus appear in most observables and in particular in the three-body recombination rate.

The derivation of the exact solution is facilitated by the fact that the operator $\hat{L}_{\varepsilon}$ does not involve any energy scale at zero energy: it is homogeneous. As a result, its action is stable in the space of power functions. The trick is then to write the exact solution as a Barnes-type contour integral over a power function where the power is the integration variable. Acting with $\hat{L}_{0}$ simply amounts to shift the integration contour. The integrand has to be chosen such that no pole is crossed when the contour is shifted back to its original position.

The solution is unique in the case of a negative scattering length where no dimer can be formed. This is in contrast with the case of a positive scattering length where the solution is not unique but can be parametrized by a single parameter. It depends on the balance between the atom-dimer and the three-particle sectors in the incoming wave. The three-body recombination rate is evaluated by canceling the incoming atom-dimer wave and then by calculating the prefactor of the outgoing atom-dimer wave. The result is an exact analytical expression that exhibits the expected logarithmic oscillations.

\section{Appendix A. Boundary condition from the two-channel model}

It is more convenient to solve Eq. (12) in Fourier space, or

$$
\left(R^{*} k^{2}+\hat{L}_{0}\right) f(\mathbf{k})=0 .
$$

The corresponding operator is rotationally invariant and thus decouples the different partial waves. For all angular momenta $l>0$, the limit $R^{*} \rightarrow 0$ can be taken directly in Eq. (A.1), and coincides with the zero range approach. The s-wave sector $l=0$, with $f(\mathbf{k})=f(k)$, requires a particular treatment in the scaling limit $R^{*} \rightarrow 0$. One notices that the operator $\hat{L}_{0}$ is homogeneous in the sense that it does not involve any scale. It implies that $\hat{L}_{0}$ acts simply on power functions, namely

$$
\hat{L}_{0} k^{-\nu-2}=G(\nu) k^{-\nu-1}
$$

where $G(\nu)$ is given by Eq. (9). This result is obtained with the change of variable $k^{\prime}=k e^{\xi}$ in Eq. (6) and the integral

$$
\int_{-\infty}^{+\infty} \frac{d \xi}{2 \pi} e^{\nu \xi}\left(\frac{e^{2 \xi}+e^{\xi}+1}{e^{2 \xi}-e^{\xi}+1}\right)=\frac{1}{\nu} \frac{\sin (\nu \pi / 6)}{\cos (\nu \pi / 2)} .
$$

Using the property Eq. (A.2), one verifies that the Barnes-type contour integral (on the right of the imaginary axis)

$$
f(k)=\int_{-i \infty}^{+i \infty} \frac{d \nu}{2 i \pi} C(\nu)\left(k R^{*}\right)^{-\nu-2}
$$


is solution of Eq. (A.1). The function

$$
C(\nu)=\frac{\pi}{\sin \left[\pi\left(\nu-i s_{0}\right)\right]} C_{+}(\nu)=\frac{\pi}{\sin \left[\pi\left(\nu+i s_{0}\right)\right]} C_{-}(\nu),
$$

such that $G(\nu) C(\nu)=-C(\nu+1)$, has no poles within the stripe $0<\operatorname{Re}(\nu)<2$ of the complex plane. Applying $\hat{L}_{0}$ on Eq. (A.4) and shifting the contour as $\nu \rightarrow \nu-1$ indeed recovers Eq. (A.1).

The large $x \gg R^{*}$ behaviour of $f(x)$ is obtained from the asymptote of $f(k)$ at small $k R^{*} \ll 1$. By closing the integration contour around the left half plane in Eq. (A.4), $f(k)$ is evaluated as a sum over terms with powers at least higher than $k^{-2}$. The poles at $\nu= \pm i s_{0}$ give the dominant contribution at small $k$. After Fourier transform, the result reads

$$
f(x) \propto \frac{\sin \left[s_{0} \ln \left(x / R_{0}\right)\right]}{x},
$$

with $R_{0}=R^{*} \exp \left[\left(\pi(\gamma+1 / 2)+\operatorname{Arg} \Gamma\left(i s_{0}\right)\right) / s_{0}\right] \simeq 0.577 R^{*}$, where $\gamma$ is given Eq. (22).

\section{Appendix B. Useful formulas}

We here list some useful relations.

First, the residue of $C_{+}$at $\nu=-i s_{0}$ is given by

$$
\operatorname{Res}\left(C_{+},-i s_{0}\right)=\frac{\Gamma\left(2+i s_{0}\right)}{\Gamma\left(1-i s_{0}\right) \Gamma\left(1+2 i s_{0}\right)} \prod_{p=1}^{\infty} \frac{\Gamma\left(-i s_{0}+u_{p}\right) \Gamma\left(1+i s_{0}+b_{p}\right)}{\Gamma\left(-i s_{0}+b_{p}\right) \Gamma\left(1+i s_{0}+u_{p}\right)},
$$

where the $p=0$ terms have been singled out from the convergent infinite product. The general identity $\Gamma(z) \Gamma(1-$ $z)=\pi / \sin (\pi z)$ is used at $z=-2 i s_{0}$ to obtain

$$
\operatorname{Res}\left(C_{+},-i s_{0}\right)=\frac{\sinh \left(2 s_{0} \pi\right)}{i \pi}\left[C_{+}\left(i s_{0}\right)\right]^{*},
$$

with the help of the property $\Gamma\left(z^{*}\right)=[\Gamma(z)]^{*}$.

Second, using the definition (20) of the functions $C_{ \pm}$, we can write

$$
C_{ \pm}(1)=\prod_{p=0}^{\infty} \frac{\Gamma\left(1+u_{p}\right) \Gamma\left(b_{p}\right)}{\Gamma\left(1+b_{p}\right) \Gamma\left(u_{p}\right)}= \pm i s_{0} \prod_{p=1} \frac{u_{p}}{b_{p}} .
$$

On the other hand, the product representation Eq. (18) of $G(\nu)$ can be evaluated at $\nu=0$ leading to

$$
G(0)=1-\frac{4 \pi}{3 \sqrt{3}}=-\left(s_{0} \prod_{p=1} \frac{u_{p}}{b_{p}}\right)^{2},
$$

with $1-4 \pi /(3 \sqrt{3}) \simeq-1.41839$. As a result, the identity

$$
C_{ \pm}(1)= \pm i \sqrt{-G(0)}
$$

is obtained.

\section{Appendix C. Saddle-point analysis at large $x$}

The integral expressions (15) and (24), for $\beta_{1}^{ \pm}$and $\beta_{2}^{ \pm}$, can be evaluated at large $x \gg a$. In contrast with the small $x$ case, the integration contour can not be closed on the right half plane. Nevertheless, the integrand exhibits rapid oscillations along the imaginary axis as $x$ is increased. A saddle-point, or stationary phase, approximation is thus carried out to extract the asymptotical behaviour.

For large $\nu \gg 1$, we have $\ln \Gamma(\nu) \simeq \ln \sqrt{2 \pi}+(\nu-1 / 2) \ln \nu-\nu$, and the coefficient $C_{+}(\nu)$ defined in Eq. (20) becomes

$$
\ln C_{+}(\nu) \simeq[\Psi(\nu)-\Psi(1-\nu)] \sum_{p=0}^{+\infty}\left(u_{p}-b_{p}\right)
$$


with the digamma function $\Psi(\nu)=\Gamma^{\prime}(\nu) / \Gamma(\nu)$. Equation (19) can be used to check that the summation over $p$ indeed converges. Since $\Psi(\nu) \simeq \ln \nu$ for $\nu \gg 1$, the limits

$$
\lim _{\nu \rightarrow \pm i \infty} C_{+}(\nu)=e^{\mp\left(i \delta_{\infty}+\pi s_{0}\right)}
$$

are obtained with the phase $\delta_{\infty}=-\pi \sum_{p=1}^{+\infty}\left(u_{p}-b_{p}\right)+\pi \simeq 1.736$ in agreement with Ref. [27]. Noting that $\sin (\pi \nu / 2) \simeq \pm i e^{\mp i \pi \nu / 2} / 2$ for $\nu \rightarrow \pm i \infty$, we rescale the integral with $\nu=x z$ and approximate

$$
\beta_{1}^{+}(x) \simeq \frac{a}{2 \sqrt{2 \pi x}} \int_{-i \infty}^{+i \infty} \frac{d z \varepsilon_{z}}{\sqrt{z}} e^{-\varepsilon_{z}\left(i \delta_{\infty}+\pi s_{0}\right)} e^{x g(z)} .
$$

We have defined $\varepsilon_{z}=\operatorname{sgn}(\operatorname{Im} z)$ and the function

$$
g(z)=z\left[\ln z-1+\ln a-\frac{i \varepsilon_{z} \pi}{2}\right] .
$$

The integral (C.3) is dominated by two symmetric saddle-points on the imaginary axis, $z_{0}^{ \pm}= \pm i / a$, derived from the condition $g^{\prime}\left(z_{0}\right)=0$. Moreover, one has $g\left(z_{0}^{ \pm}\right)=\mp i / a$ and $g^{\prime \prime}\left(z_{0}^{ \pm}\right)=\mp i a$. After integration over the quadratic fluctuations around the two saddle-points, the asymptotic form

$$
\beta_{1}^{+}(x) \simeq-i \frac{a}{2}\left(e^{\pi s_{0}} e^{i \delta_{\infty}} \frac{e^{i x / a}}{x}-e^{-\pi s_{0}} e^{-i \delta_{\infty}} \frac{e^{-i x / a}}{x}\right)
$$

is obtained for $x \gg a$. The same result is derived for $\beta_{1}^{-}(x)$ with $s_{0}$ replaced by $-s_{0}$. In this asymptotic region $x \gg a$, Eq. (C.5) describes a combination of incoming and outgoing atom-dimer waves.

The calculation is similar for the case of $\beta_{2}^{ \pm}$. The difference is expressed by the following limit

$$
\frac{\sin (\pi \nu / 2)}{\tan (\pi \nu)} \simeq \frac{e^{\mp i \pi \nu / 2}}{2}
$$

for $\nu \rightarrow \pm i \infty$. The final result reads

$$
\beta_{2}^{+}(x) \simeq \frac{a}{2}\left(e^{\pi s_{0}} e^{i \delta_{\infty}} \frac{e^{i x / a}}{x}+e^{-\pi s_{0}} e^{-i \delta_{\infty}} \frac{e^{-i x / a}}{x}\right),
$$

with $s_{0} \rightarrow-s_{0}$ for $\beta_{2}^{-}(x)$. Again incoming and outgoing waves coexist in this asymptotic form.

\section{References}

[1] E. Braaten, H.-W. Hammer, Universality in few-body systems with large scattering length, Phys. Rep. 428 (5-6) (2006) 259-390.

[2] I. Bloch, J. Dalibard, W. Zwerger, Many-body physics with ultracold gases, Rev. Mod. Phys. 80 (3) (2008) 885-964.

[3] E. Braaten, A. Nieto, Quantum corrections to the energy density of a homogeneous Bose gas, Eur. Phys. J. B 11 (1) (1999) $143-159$.

[4] P. F. Bedaque, H.-W. Hammer, U. van Kolck, Renormalization of the three-body system with short-range interactions, Phys. Rev. Lett. 82 (3) (1999) 463-467.

[5] E. Braaten, H.-W. Hammer, T. Mehen, Dilute bose-einstein condensate with large scattering length, Phys. Rev. Lett. 88 (4) (2002) 040401.

[6] D. S. Petrov, C. Salomon, G. V. Shlyapnikov, Weakly bound dimers of fermionic atoms, Phys. Rev. Lett. 93 (9) (2004) 090404

[7] C. Mora, A. Komnik, R. Egger, A. O. Gogolin, Four-body problem and bec-bcs crossover in a quasi-one-dimensional cold fermion gas, Phys. Rev. Lett. 95 (8) (2005) 080403.

[8] E. Nielsen, J. H. Macek, Low-energy recombination of identical bosons by three-body collisions, Phys. Rev. Lett. 83 (8) (1999) 1566-1569.

[9] B. D. Esry, C. H. Greene, J. P. Burke Jr, Recombination of three atoms in the ultracold limit, Phys. Rev. Lett. 83 (9) (1999) 1751-1754.

[10] P. F. Bedaque, E. Braaten, H. W. Hammer, Three-body recombination in Bose gases with large scattering length, Phys. Rev. Lett. 85 (5) (2000) 908-911.

[11] D. S. Petrov, Three-boson problem near a narrow feshbach resonance, Phys. Rev. Lett. 93 (14) (2004) 143201.

[12] T. Köhler, K. Göral, P. S. Julienne, Production of cold molecules via magnetically tunable feshbach resonances, Rev. Mod. Phys. 78 (4) (2006) 1311-1361.

[13] L. Platter, Low-Energy Universality in Atomic and Nuclear Physics, Few-Body Syst. 46 (3) (2009) $139-171$.

[14] J. P. D'Incao, B. D. Esry, Scattering length scaling laws for ultracold three-body collisions, Phys. Rev. Lett. 94 (21) (2005) 213201. 
[15] V. Efimov, Weakly-bound states of three resonantly-interacting particles, Yad. Fiz 12 (1970) 1080, [Sov. J. Nucl. Phys 29, 589 (1971)].

[16] T. Kraemer, M. Mark, P. Waldburger, J. G. Danzl, C. Chin, B. Engeser, A. D. Lange, K. Pilch, A. Jaakkola, H. C. Nägerl, R. Grimm, Evidence for Efimov quantum states in an ultracold gas of caesium atoms, Nature 440 (7082) (2006) $315-318$.

[17] S. Knoop, F. Ferlaino, M. Mark, M. Berninger, H. Schöbel, H. C. Nägerl, R. Grimm, Observation of an Efimov-like trimer resonance in ultracold atom-dimer scattering, Nature Physics 5 (3) (2009) 227-230.

[18] M. Zaccanti, B. Deissler, C. D’Errico, M. Fattori, M. Jona-Lasinio, S. Müller, G. Roati, M. Inguscio, G. Modugno, Observation of an Efimov spectrum in an atomic system, Nature Physics 5 (8) (2009) 586-591.

[19] G. Barontini, C. Weber, F. Rabatti, J. Catani, G. Thalhammer, M. Inguscio, F. Minardi, Observation of heteronuclear atomic efimov resonances, Phys. Rev. Lett. 103 (4) (2009) 043201.

[20] N. Gross, Z. Shotan, S. Kokkelmans, L. Khaykovich, Observation of universality in ultracold ${ }^{7}$ li three-body recombination, Phys. Rev. Lett. 103 (16) (2009) 163202.

[21] T. B. Ottenstein, T. Lompe, M. Kohnen, A. N. Wenz, S. Jochim, Collisional stability of a three-component degenerate fermi gas, Phys. Rev. Lett. 101 (20) (2008) 203202

[22] J. H. Huckans, J. R. Williams, E. L. Hazlett, R. W. Stites, K. M. O'Hara, Three-body recombination in a three-state fermi gas with widely tunable interactions, Phys. Rev. Lett. 102 (16) (2009) 165302.

[23] S. Pollack, D. Dries, R. Hulet, Universality in Three-and Four-Body Bound States of Ultracold Atoms, Science 326 (5960) (2009) 1683.

[24] E. Braaten, H.-W. Hammer, Efimov physics in cold atoms, Ann. Phys. (N.Y.) 322 (1) (2007) $120-163$.

[25] E. Nielsen, D. V. Fedorov, A. S. Jensen, E. Garrido, The three-body problem with short-range interactions, Phys. Rep. 347 (5) (2001) 373-459.

[26] G. Gasaneo, S. Ovchinnikov, J. Macek, Kontorovich-Lebedev representation for zero-range potential eigensolutions, J. Phys. A 34 (2001) 8941-8954.

[27] J. H. Macek, S. Ovchinnikov, G. Gasaneo, Solution for boson-diboson elastic scattering at zero energy in the shape-independent model, Phys. Rev. A 72 (3) (2005) 032709.

[28] J. H. Macek, S. Yu Ovchinnikov, G. Gasaneo, Exact solution for three particles interacting via zero-range potentials, Phys. Rev. A 73 (3) (2006) 032704.

[29] G. V. Skorniakov, K. A. Ter-Martirosian, The Three-Body Problem with Short-Range Forces. Scattering of Low-Energy Neutrons by Deuterons, Zh. Eksp. Teor. Fiz. 31 (1956) 775, [Sov. Phys. JETP 4, 648 (1957)].

[30] A. O. Gogolin, C. Mora, R. Egger, Analytical solution of the bosonic three-body problem, Phys. Rev. Lett. 100 (14) (2008) 140404 .

[31] D. S. Petrov, Three-body problem in fermi gases with short-range interparticle interaction, Phys. Rev. A 67 (1) (2003) 010703.

[32] C. Mora, R. Egger, A. O. Gogolin, A. Komnik, Atom-dimer scattering for confined ultracold fermion gases, Phys. Rev. Lett. 93 (17) (2004) 170403.

[33] C. Mora, R. Egger, A. O. Gogolin, Three-body problem for ultracold atoms in quasi-one-dimensional traps, Phys. Rev. A 71 (5) (2005) 052705

[34] G. S. Danilov, On the Three-Body Problem with Short-Range Forces, Zh. Eksp. Teor. Fiz. 40 (1961) 498, [Sov. Phys. JETP $13,349(1961)]$.

[35] L. H. Thomas, The interaction between a neutron and a proton and the structure of ${ }^{3} \mathrm{~h}, \mathrm{Phys} . \mathrm{Rev} .47$ (12) (1935) $903-909$.

[36] R. A. Minlos, L. D. Faddeev, Comment on the Problem of Three Particles with Point Interactions, Zh. Eksp. Teor. Fiz. 41 (1961) 1850, [Sov. Phys. JETP 14, 1315 (1962)].

[37] R. A. Minlos, L. D. Faddeev, On the point interaction for a three-particle system in quantum mechanics, Doklady Akademii Nauk SSR 141 (1961) 1335, [Sov. Phys. Doklady 6, 1072 (1962)].

[38] E. Nielsen, H. Suno, B. D. Esry, Efimov resonances in atom-diatom scattering, Phys. Rev. A 66 (1) (2002) 012705.

[39] E. Braaten, H.-W. Hammer, M. Kusunoki, Universal equation for efimov states, Phys. Rev. A 67 (2) (2003) 022505.

[40] D. V. Fedorov, A. S. Jensen, Regularization of a three-body problem with zero-range potentials, J. Phys. A 34 (2001) 6003.

[41] K. Helfrich, H.-W. Hammer, D. S. Petrov, Three-body problem in heteronuclear mixtures with resonant interspecies interaction, Phys. Rev. A 81 (4) (2010) 042715.

[42] J. P. D'Incao, B. D. Esry, Enhancing the observability of the Efimov effect in ultracold atomic gas mixtures, Phys. Rev. A 73 (3) (2006) 30703

[43] D.S. Petrov, unpublished results (2005). 